NASA Technical Memorandum 88923

AIAA-87-0283

\title{
Two- and Three-Dimensional Viscous Computations of a Hypersonic Inlet Flow
}

William G. Kunik and Thomas J. Benson

Lewis Research Center

Cleveland, Ohio

and

Wing-Fai Ng and Arthur Taylor

Virginia Polytechnic Institute and State University

Blacksburg, Virginia

Prepared for the

25th Aerospace Sciences Meeting

sponsored by the American Institute of Aeronautics and Astronautics

Reno, Nevada, January 12-15, 1987 
TWO- AND THREE-DIMENSIONAL VISCOUS COMPUTATIONS

OF A HYPERSONIC INLET FLOW

\author{
William G. Kunik and Thomas J. Benson \\ National Aeronautics and Space Administration \\ Lewis Research Center \\ Cleveland, Ohio 44735
}

and

Wing-Fai $\mathrm{Ng}$ and Arthur Taylor

Virginia Polytechnic Institute and State University

Blacksburg, Virginia 24061

\title{
SUMMARY
}

A three-dimensional parabolized Navier-Stokes code has been used to investigate the flow through a Mach 7.4 inlet. A two-dimensional parametric study of grid resolution, turbulence modeling and effect of gamma has been done and compared with experimental results. The results show that mesh resolution of the shock waves, real gas effects and turbulence length scaling are very important to get accurate results for hypersonic inlet flows. In addition, a threedimensional calculation of the Mach 7.4 inlet has been done on a straight sideplate configuration. The results show that the glancing shock/boundary layer interaction phenomena causes significant three-dimensional flow in the inlet.

\section{INTRODUCTION}

Recent recommendations from the Aeronautical Policy Review Committee (under the direction of the White House Office of Science and Technology) have revived national interest in hypersonic propulsion. Much of the technology base for this effort is expected to develop from the progressive supersonic and hypersonic advances in computational fluid mechanics. Several factors affecting the accuracy of hypersonic inlet calculations are studied in the current investigation by comparing the results of two-dimensional viscous calculations with experimental results.

Hypersonic flows are characterized by high Mach numbers in the inviscid regions, high gradients in the vicinity of shock waves or solid surfaces, and thick, heated boundary layers. To accurately model the high gradients near shocks and along surfaces, one must employ large numbers of mesh points in the calculation. Most flow calculations in this regime encounter some degree of post-shock flow oscillations, which can be minimized either through some form of artificial viscosity, upwind differencing techniques, or increased grid resolution. To accurately model the high temperatures found in hypersonic flows, one needs to include real-gas effects in the energy equation models. This model can take several forms from the simplest specific heats as a function of temperature to the more complex chemical kinetic and specie models. To accurately model the thick boundary layers present in hypersonic flows, one may need to consider a variety of turbulence and transition models. In the present study, a supersonic parabolized Navier-Stokes solver has been used to investigate some of these problems. 
Computer code modelling of the flow in supersonic and hypersonic inlets has increased recentiy due to improved computer algorithms and more powerful computers such as the Cray-XMP and Cyber 205. Some researchers (refs. 1 to 3), have used the unsteady Reynolds-averaged Navier-Stokes (NS) equations marched in time to a steady state solution to describe the flow in the inlet. At NASA Lewis, the inlet has been analyzed with a zonal methodology (ref. 4); NS codes are used only in he transonic terminal shock region, while higher speed parabolized Navier-Stokes (PNS) codes are used in the supersonic and subsonic diffusers. Through an extensive code verification effort (ref. 4), it has been found that the higher computational speed of the PNS codes provides the anaiyst sufficient grid resoiution to resolve flow phenomenon and that lack of sufficlent grid resolution often leads to erroneous results (ref. 5). To attempt the same levels of grid resolution with an NS solver as is available to a PNS solver would require orders of magnitude greater computing time due to the time-dependence of the NS solution and due to restrictions on the magnitude of the time increment relative to the spatial grid size.

An acknowledged dissadvantage of the PNS solver for high speed flows is the fallure of the solver when large regions of subsonic or reverse flow are encountered. The failure of a PNS analys is to accurately model large regions of separated flow in a mixed-compression inlet may not be a serious problem, however, because such flows will trigger an inlet unstart. In an inlet unstart, a normal shock is formed inside the inlet by the flow separation and this shock moves very quickly upstream. The oblique shock structure with in the inlet is destroyed creating subsonic flow throughout the inlet. The performance of the inlet changes dramatically since the flow becomes subsonic through a very strong normal shock instead of the multiple obliques and weak normal shock of a well-designed mixed-compression inlet. Mixed compression inlets are never designed with large regions of subsonic or reverse flow because of the inlet unstart problem. It is necessary in the analysis of mixed compression inlets to accurately predict the occurence of reverse flows which trigger unstarts, but it is not necessary to calculate the details of the recirculation.

The three-dimensional supersonic viscous marching analysis used in this study solves the PNS equations for supersonic flow by an efficient linearized block implicit scheme (ref. 6). The code has been extensively verified at NASA Lewis, with particular emphasis on the calculation of the glancing shock/boundary layer interaction (GSBLI) (ref. 7). These studies have demonstrated the code's ability to properly model the complex three-dimensional phenomena present in this interaction. It also uncovered the importance of, and established the levels of grid resolution necessary to model this interaction. Besides demonstrating the code's ability to match the experimental data, these studies have increased our understanding of the physical processes present in these interactions and their effects on inlet performance. This interaction is very important in the three-dimensional calculations presented here.

\section{TWO-DIMENSIONAL RESULTS}

Several calculations have been made of the flow through a Mach 7.4 inlet and the results of the calculations have been compared to the experimental results. The hypersonic inlet to be calculated was originally tested at NASA Ames and the experimental results are documented in reference 8 . A schematic drawing of one configuration of the inlet is shown in figure 1 . At Mach 7.4 
the nominal $6.5^{\circ}$ wedge produces a Mach 6.0 flow at the entrance to the inlet. A shock is generated by the cowl lip and this shock traverses the internal flow passage and is reflected by the ramp surface. In the vicinity of the shock reflection, the flowfield was surveyed with a traversing pitot pressure probe to obtain pressure profiles throughout the inlet. By varying the grid resolution, turbulence models, and ratio of specific heats in the calculations, the sensitivity to these factors can be assessed. These answers can then aid future calculations in this speed regime and beyond.

Figures 2 to 4 show some results from calculations of the inlet. In each figure a schematic of the inlet is shown at the top and a comparison of the calculated and experimental pitot pressure profiles is shown at the bottom. The location of the profiles are noted on the upper schematic. In all cases the results of the analysis are given by solid or dashed lines, while the experimental results are noted by the circles. In all of the calculations, the free stream conditions were set to the experimental tunnel conditions of Mach number equal to 7.4 , total pressure equal $4.14 \times 10^{6} \mathrm{~N} / \mathrm{m}^{2}$ and total temperature equal to $811 \mathrm{~K}$. A 11 of the calculations were run fully turbulent, although there was some indication in the test that transition occurs on both ramp and cowl surfaces.

Figure 2 shows a comparison of two calculations with the experimental pitot profile. Considering the profile at the left, the lower part of the figure shows the thick boundary layer which has grown on the ramp surface; the thickness is indicated by the location where the curve turns vertical. Continuing up the curve, the sharp turn to the right is indicative of the cowl shock location while the gradual rise to the right and up is caused by the distributed compression on the inside of the cowl. Near the top of the curve, it turns quickly back to the left which indicates the thickness of the boundary layer on the cowl. In general, the comparison between analysis and experiment is excellent; the shock location and strength has been correctly modelled, as has the thickness of the ramp boundary layer. The boundary layer on the cowl is thinner than the calculated results because of laminar to turbulent transitioning effects.

The two calculations of figure 2 have been run with different numbers of mesh points in the direction normal to the ramp and cowl surfaces. When one hundred mesh point are used, the flow experiences severe post-shock pressure oscillations which are most evident in the middle profile dashed curve. Other researchers have experienced these oscillations in high speed flow calculations particularly when central differencing techniques are used. While others are attempting to overcome these numerical problems with special differencing schemes, or various types of artificial damping, this study indicates that the problem can be overcome with increased mesh resolution. This is shown by the smooth solid curves on figure 2, which used three hundred mesh points in the normal direction. Adequate control of the post-shock oscillations was obtained using a minimum of two hundred radial mesh points. The resulting mesh of two hundred thousand mesh points took approximately three minutes of CPU time on the Lewis CRAY X-MP.

Figure 3 shows the results of two calculations employing different length scales in the turbulence model. The dashed line shows the results using a McDonald-Camarata model which has been successfully used in the PNS code for 
many different types of problems in the low supersonic speed regime (refs. 5, 7, and 9). For the hypersonic calculations, however, this model breaks down. Bushnell and Beckwith found similar problems with this model in their earlier research (ref. 10), and using a length scale model based on this work, the results appear as the solid itne. The comparison with experiment is much better and the improved model is recommended for calculation of hypersonic flows. This calculation indicates possible difficulties in the calculation of high speed flows for which no comparison with experimental data is avallable; a turbulence model which works quite well at lower speeds gives erroneous results for higher Mach number flows.

Figure 4 shows two calculations of the Mach 7.4 inlet with different vaiues of the ratio of specific heats. The dashed line was calculated at gamma equal to 1.4 , while the solid line was calculated at 1.38 . A rather small change in this parameter has produced a large change in the pitot profiles. At the higher value of gamma, the shock from the cowl has moved upstream and become stronger. This figure indicates two important points; first, real gas effects which can vary the local value of gamma will have first order effects on the accurate calculation of the flow in high speed inlets, and second, when testing inlets in facilities, it will be necessary to match fiight Mach number, temperature and gamma to obtain meaningful results. Testing hypersonic inlets in rocket exhaust nozzles, for example, may match flight Mach number and temperature conditions, yet fall to match gamma because of the combustion by-products present. This calculation shows that small differences in the value of gamma can lead to large differences in the assessment of inlet performance

\section{THREE-DIMENSIONAL RESULTS}

Once the two-dimensional parametric study was completed, it was decided that the inlet should be examined three-dimensionally to determine the effect of the sideplates on the internal flowfield. Previous calculations of a Mach 5.0 inlet (ref. 11), and benchmark studies of GSBLI (refs. 7 and 9), indicate strong three-dimensional effects occur in rectangular inlet with sideplates. The experiment did not provide sufficient instrumentation for detailed study of three-dimensional effects. Figure 5(a) shows a schematic of the probe locations in the spanwise direction of the inlet. In the internal passage of the inlet the surveys were taken close to the centerline and therefore major threedimensional effects would not have been detected. The axial locations are shown in figure 4. Some qualitative comparison with the throat rake data will be given.

The three-dimensional calculation employed a computational mesh of one hundred mesh points in the $Y$ direction and fifty points in the $Z$ direction (the coordinate axes are shown in fig. 5(b)). Although the two-dimensional results indicated that more points are needed to control the post shock oscillations, only the overall effect of the sideplates on the two-dimensionally of the flow was to be examined. To simplify the calculations, the sideplate in the calculation begins at the cowl lip and is perpendicular to the ramp surface. In the experiment, the sideplate was cut back as shown in figure $5(b)$; the calculation does not model this cut back.

Figures 6 to 10 shown Mach number contours and secondary velocity vectors at vartous $Y-Z$ planes in the internal portion of the inlet. The bottom of the figures show the flowfield in a cross section of the inlet; the ramp surface 
is on the bottom, the cowl surface is on the top, and the sideplates are on both sides. Because of flow symmetry, only half of the inlet was calculated. The side view of the inlet is shown at the top of each figure indicating with a vertical line the relative location of the plane in the inlet. The axial location given with the plot, $X / H C$, is referenced to the inlet capture height. A scale in shown on the left hand side for the Mach number contours and on the right hand side a reference vector of one-tenth of the free stream velocity is shown. Boundary layers are noted by the concentration of Mach contours near a solid surface and shock waves are shown as a concentration of Mach contours away from a solid surface. Shock waves are also noted in the secondary velocity vectors when the direction of the vectors change abruptly.

In Figure 6 the contours are shown just upstream of the cowl 1ip. The Mach number contours show that the centerbody shock wave has passed out of the flow field. and that a thick boundary layer has developed on the centerbody surface. The secondary velocity vectors indicate that the flow is uniform in the 2-direction. 011 flow tests in reference 8 show that the flow at the cowl lip had been turned nearly six degrees in the Z-direction because of edge effects on the experimental wedge. The analys is has assumed an infinite wedge and does not predict this outflow. Figure 7 shows a cross section between the cowl lip and throat where the cowl shock wave is moving toward the centerbody surface. The figure shows that a vortex is forming on the back side of the shock wave as it glances across the sideplate boundary layer; this interaction was verified in reference 7 . The vortex is pumping the low energy fluid in the cowl boundary layer down along the sideplate to the ramp surface. The Mach number contours show this by the thickening of the sideplate boundary layer on the back side of the shock wave. Figure 7 also shows that a weak shock is moving out away from the sideplate in the Mach number contours, as noted by the biip in the contours on the cowl surface and in the shock wave. This is developed from the boundary layer growth along the sideplate. The shock wave near the sideplate is skewed toward the ramp surface as indicated in both plots.

Figure 8 shows the Mach number and secondary velocity plots at the point where the cowl shock wave reflects with the ramp surface. The vortex formed by the GSBLI has strengthened and is now pumping fluid along the ramp surface toward the centerline of the inlet. The boundary layer in the lower corner has thickened much more than in the rest of the cross section. The effect of the strong vortex is seen in the boundary layer along the ramp surface by its thickening to about the 50 percent point in the spanwise direction. At this point the three-dimensional effects of the sideplates have not reached the survey plane point which is at approximately the 30 percent point from the centerline. At this point in the design the cowl shock wave should have canceled on the ramp surface. With the thick boundary layers on all the surfaces and the skewing of the shock wave near the sideplate, the shock wave does not cancel at this point. The contours of figure 9 are at the point where the shock wave has start to reflect on the cowl surface. The vortex shown in the secondary velocity vectors indicate that it is still very strong. The vortex is still pumping low energy fluid into the lower corner. The boundary layer shown by the Mach number contours is very distorted in the lower corner and along the ramp surface. The ramp boundary layer thins toward the centerline of the inlet. The secondary velocity vectors show crossflow velocities near the internal passage survey plane. Figure 10 shows the contours near the inlet exit. At this point the flow field is highly distorted and the shock wave is 
bent across the width of the cross section. The vortex due to the GSBLI is situated in the lower corner and it is still very strong. The pumping effect of the vortex is being felt over approximately 75 percent of the cross section.

Figure 11 shows the static pressure contours along the cowl and the centerbody surfaces. Both plots are from the sideplate, which are on the upper part of both plots, to the centerline of the inlet, which are on the lower part of both plots. Along the centerbody surface the cowl shock wave impingement is shown by the first concentration of contour lines. At this point the shock is two-dimensional over about 60 percent of the width of the inlet, but at the next shock impingement on the centerbody the shock wave is skewed over almost the entire width of the inlet. Also, after both shock reflections on the centerbody an overpressure region is formed away from the centerline as was seen in the GSBLI case of reference 9. The static pressure contours on the cowl surface show that the sideplate is generating a weak shock wave which is distorting the distributive compression on the front part of the cowl. The centerbody shock impingement shows the same effects as on the centerbody sur-: face. A large portion of the flow field at this point is being affected by the interaction of the cowl shock and sideplate boundary layer.

In figure 12 contour plots of static pressure are shown in $X-Y$ planes, at selected spanwise locations. The four planes are at; (1) the centerline $(Z=0)$; (2) the internal survey plane $(Z=0.3) ;(3)$ the 50 percent span poin $(Z=0.5)$; and $(4)$ the 75 percent span point $(Z=0.75)$. The centerline plane shows the same shock wave pattern as the two-dimensional results. At the internal passage survey plane the shock pattern is very similar to the centerline shock pattern. The only major difference is near the last shock reflection on the centerbody, where the effect of the sideplate causes the shock impingement point to move upstream. At the 50 percent span plane the progression of the shock pattern upstream is seen after the first reflection on the centerbody. The shock wave generated by the cowl lip does not show much change from the centerline shock. The final plane shows the first centerbody shock reflection point moves upstream of the reflection point at 50 percent. The reflected shock is more highly inclined to the ramp and strikes the cowl further upstream. This change in the shock wave pattern with spanwise location may help explain a phenomenon noted in the experimental report. A Schlierin photograph of this region shows the cowl shock striking the centerbody and two waves reflecting back towards the cowl. The computational results indicate that this may be due to the curvature of the shock wave seen in figure 12 . This does not affect the cowl shock wave because the three-dimensionally of the flow field has not developed enough at this point and it intensifies after the shock wave boundary layer interaction on the ramp surface.

The three-dimensional flow features seen in the previous figures cause the performance of this inlet to be less than predicted two-dimensionally. Figure 13 shows pitot pressure contours at the exit of the inlet as calculated by the three-dimensional analysis. Only half of the inlet is shown in this figure, with the sidewall at the left. The GSBLI has caused the pitot contours to be highly distorted in the corner formed by the ramp and sidewall. The effects of the interaction are seen to extend over more than 50 percent of the cross section. The instrumentation of the experiment was concentrated along the centerline except near the throat. Figure 14 shows a comparison of the analysis and experimental results in this region. The comparison can only be made qualitatively because of insufficient information in the experimental 
report. However, the figure shows that both analysis and experimental ind icate strong vartations in pitot pressure with span; and that both indicate higher pitot pressures near the cowl surface near the sidewall.

\section{CONCLUSIONS}

From the two-dimensional results presented here three major conclusions can made. First, to adequately model the shock wave structure and to eliminate post shock oscillations in high speed flow fields a large number of mesh points are required. The results of this study indicate that two hundred points in the $Y$-direction are adequate to control post shock oscillations in this analysis. The increased number of mesh points can still be calculate very quickly with the analysis. Other researchers have indicated that the same results can be obtained by using alternate differencing techniques. Second, to model the boundary layer growth in these flows, which are very large, proper scaling of the turbulence is important. Very good agreement with the experimental data is obtained using a mixing length model with proper corrections for high speed flow. Third, real gas effects will become very important in accurately predicting the correct pressure distributions in high speed, high temperature flows. The results presented here show that changing gamma over the entire flow field dramatically change the free stream pressure levels. A more sophisticated model for real gas effects should be incorporated later.

The three-dimensional calculation presented in this report have led to the following conclusions. The glancing shock/boundary layer interaction produces a strong vortex that persists throughout the internal passage of the inlet. This vortex redistributes the boundary layer into the lower corner of the inlet and then proceeds to distort the ramp boundary layer. To achieve a canceled shock wave in the inlet, the effect of the boundary layers on the shock wave interaction at solid boundaries must be taken into account. Also, the effect of the sideplates on the shock wave necessitates that inlet design must be based on an understanding of the three-dimensional nature of the flow field.

\section{REFERENCES}

1. White, M.E.; Drummond, J.P.; and Kumar, A.: Evolution and Status of CFD Techniques for Scramjet Appilications. AIAA Paper 86-0160, Jan. 1986.

2. Knight, D.D.: Improved Numerical Simulation of High Speed Inlets Using the Navier-Stokes Equations. AIAA Paper 80-0383, Jan. 1980.

3. Campbe11, A.F.; and Forester, C.K.: Evaluation of a Method for Analyzing the Aperture Region of Two-Dimensional External Compression In lets. AIAA Paper 85-3072, Oct. 1985.

4. Anderson, B.H.: Three-Dimensional Viscous Design Methodology for Advanced Technology Aircraft Supersonic Inlet Systems. AIAA Paper 84-0194, Jan. 1984 .

5. Benson, T.J.; and Anderson, B.H.: Validation of a Three-Dimensional Viscous Analysis of Axisymmetric Supersonic Inlet Flow Fields. AIAA Paper 83-0137, Jan. 1983. 
6. Buggeln, R.C., et al.: Development of a Three-Dimensional Supersonic Inlet flow Analysis. NASA CR-3218, 1980.

7. Anderson, B.H.; and Benson, T.J.: Numerical Solution to the Glancing Sidewal1 Oblique Shock Wave/Turbulent Boundary Layer Interaction in Three Dimension. AIAA Paper 83-0136, Jan. 1983.

8. Gnos,A.V., et al.: Investigation of Flow Fields within Large-Scale Hypersonic Inlet Models. NASA TN D-7150, 1973.

9. Benson, T.J.; and Anderson, B.H.: A Study of Three Dimensional Shock Wave Turbulent Boundary Layer Interactions Present Within Aircraft Inlets. AIAA Paper 84-1558, June 1984.

10. Bushne11,D.M.; and Beckwith, I.E.: Calculation of Nonequilibrium Hypersonic Turbulent Boundary Layers and Comparison with Experimental Data. AIAA J., Vo1. 8, No. 8, Aug. 1970, pp. 1462-1469.

11. Benson, T.J.: Three-Dimensional Viscous Calculation of Flow in a Mach 5.0 Hypersonic Inlet. AIAA Paper 86-1461, June 1986. 


\section{ORIGINAL PAGE IS OF. POOR QUALTY}

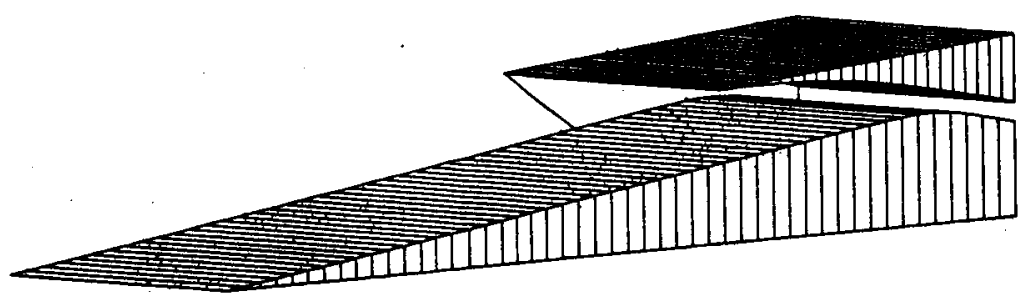

FIGURE 1. - MACH 7.4 HYPERSOHIC INLET GEORETRY.

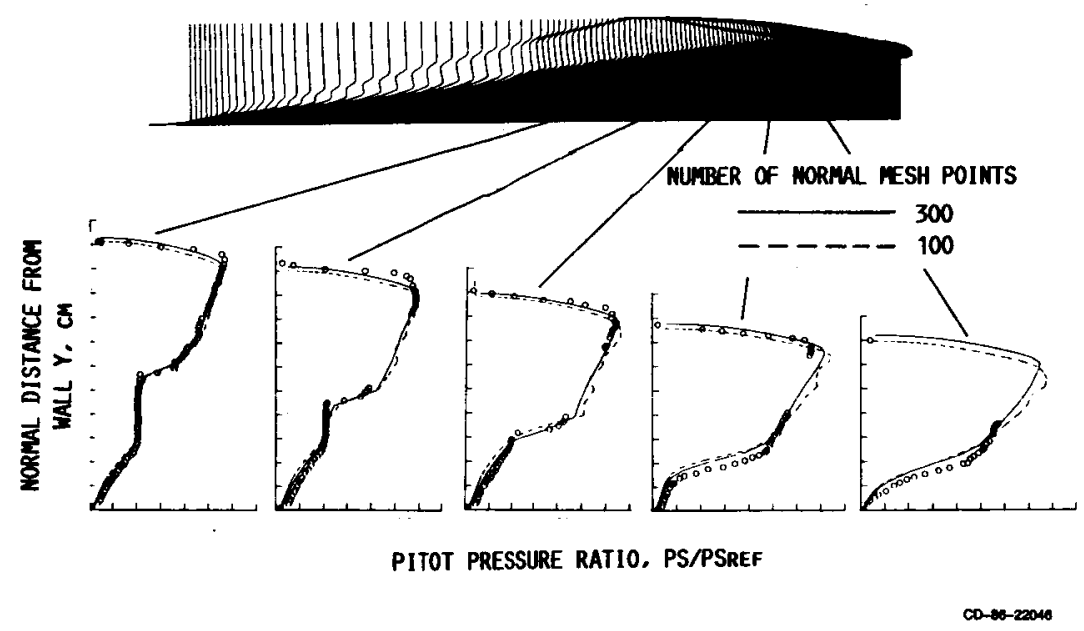

FIGURE 2. - EFFECT OF GRID RESOLUTION ON IMLET CALCULATION. 


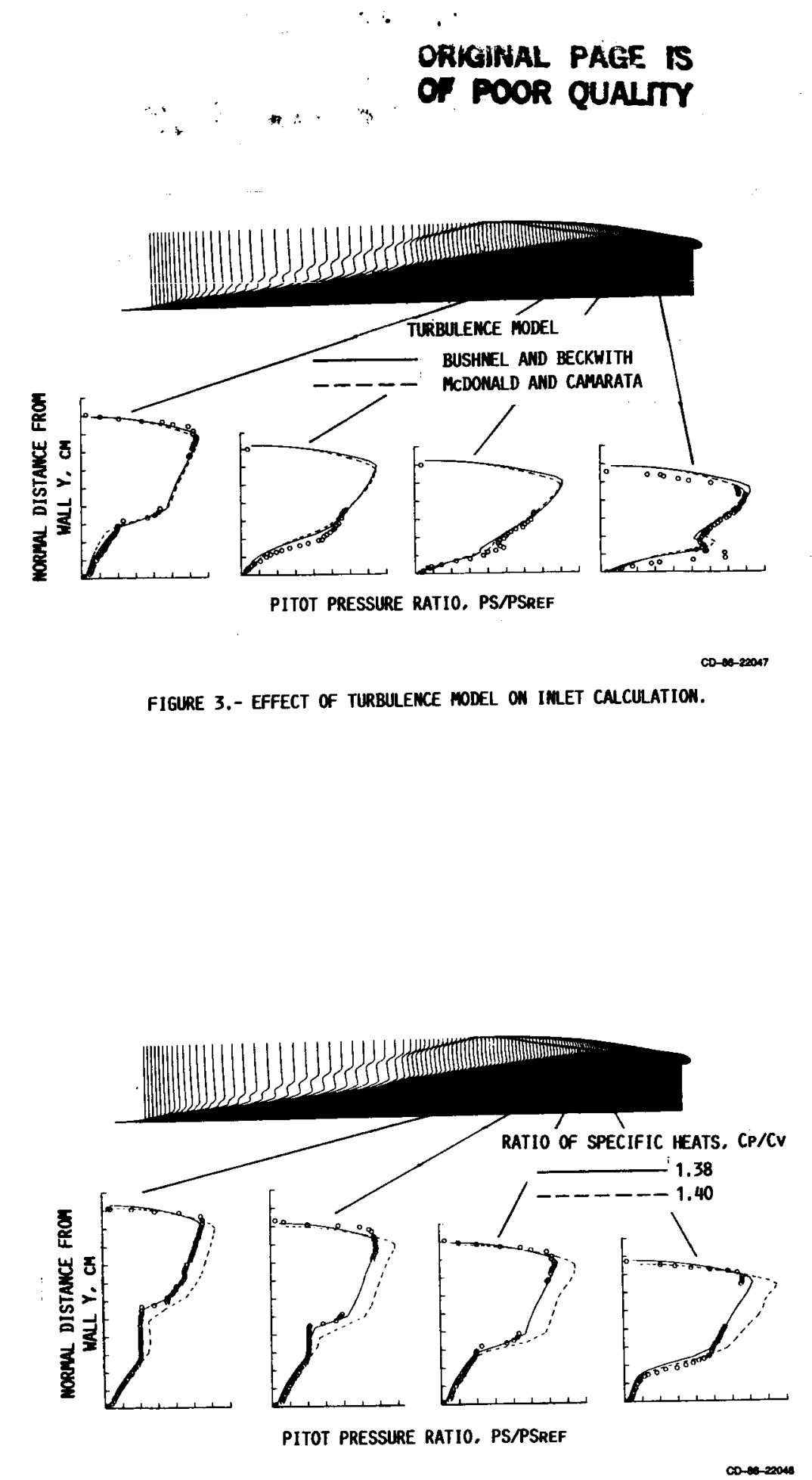

FigURE 4. - EFFECT OF SPECIFIC HEAT RATIO ON IMLET CALCULATION. 


\section{ORIGINAL PAGE IO \\ OF. POOR QUALITY}

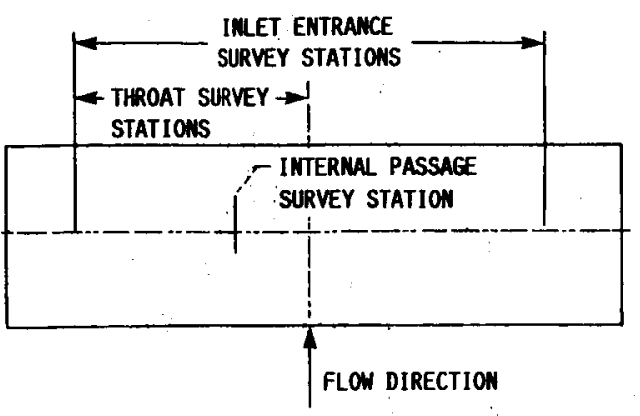

(A) PROBE LOCATIONS-SPANISE VIEW.

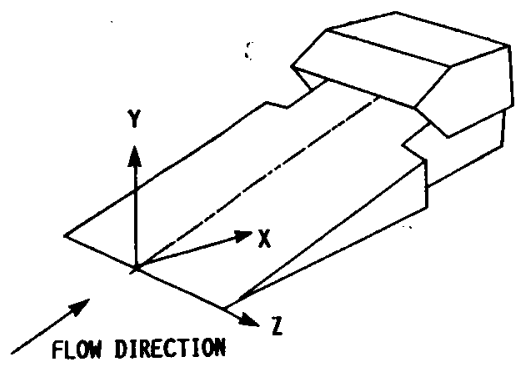

(B) 3D SCHEMATIC OF P8 IMLET.

FIGURE 5. - LATERAL SURVEY LOCATIONS AND SCHEMATIC SCHEMATIC OF IMLET.

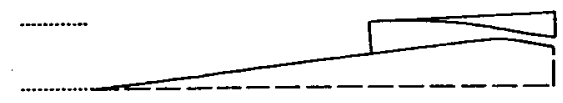

$\begin{array}{cc}\text { STATION } & 519 \\ \text { X/KC } & 0.5402 \mathrm{E} 01\end{array}$
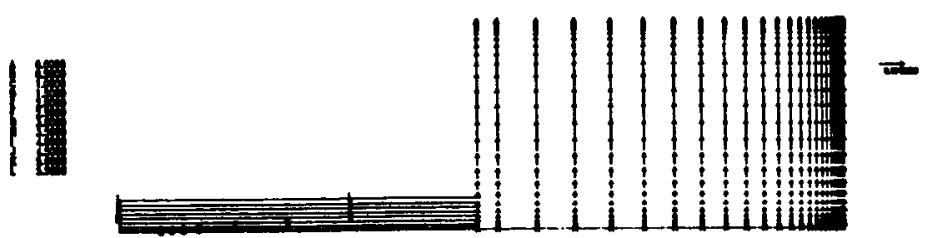

FIGURE 6. - MACH MUMBER CONTOURS AND SECONDARY VELOCITY VECTORS BEFORE COML LIP. 


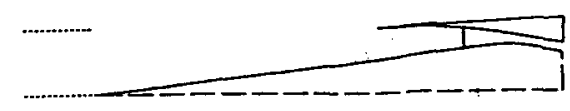

$\begin{array}{cc}\text { STATION } & 1039 \\ X / H C= & 0.6710 E 01\end{array}$

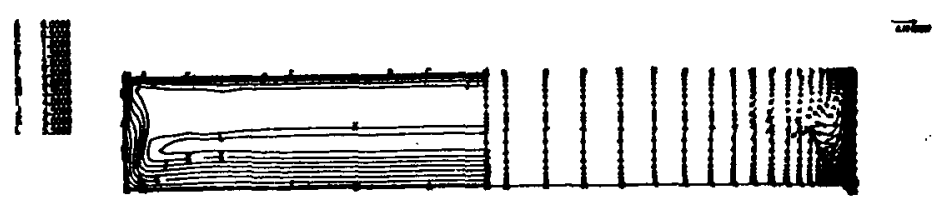

FIGURE 7. - MACH MUMBER CONTOURS AND SECONBARY VELOCITY VECTORS DOWMSTREAM of COML LIP.

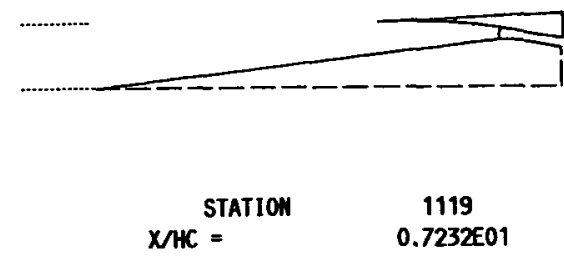

|

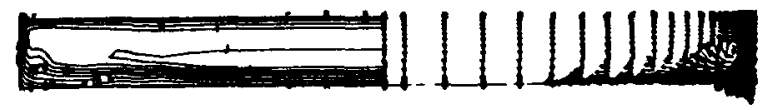

FIGURE 8. - MACH MUMBER CONTOURS AND SECONDARY VELOCITY VECTORS AT CENTERBODY SHOCK REFLECTION. 


\section{ORIGINAL PAGE IS
OF POOR QUALITY}

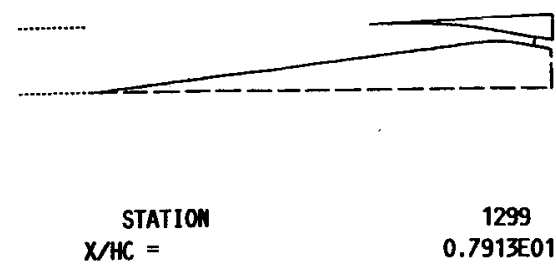

II

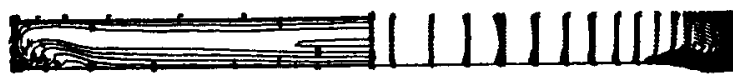

FIGURE 9. - MACH MUMBER CONTOURS AND SECONDARY VELOCITY VECTORS AT COML SHOCK REFLECTION.

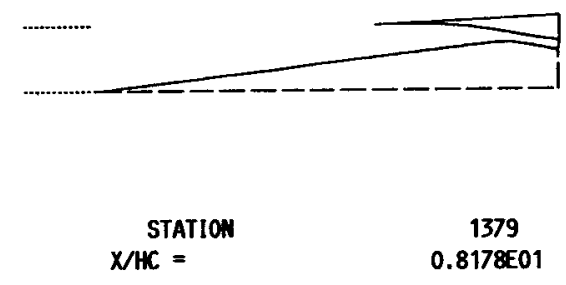

II

$\longrightarrow$

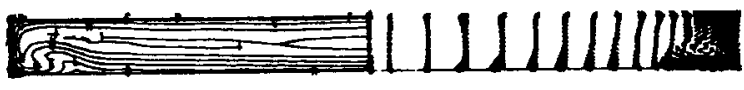

FIGURE 10. - MACH MLYBER CONTOURS AND SECONDARY VELOCITY VECTORS AT THE IMLET EXIT. 


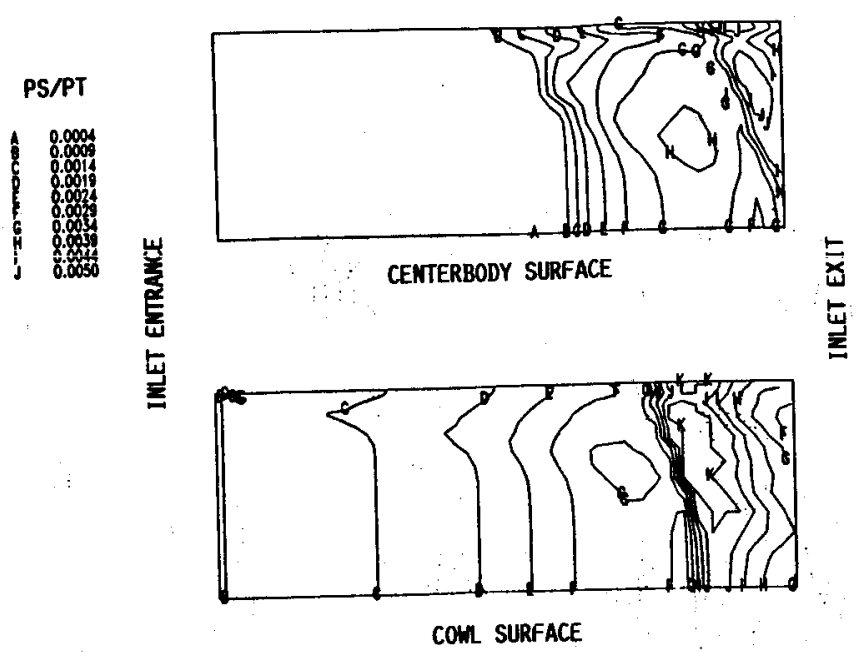

FIGURE 11.- SURFACE STATIC PRESSURE DISTRIBUTIONS ON THE CENTERBODY AND THE COHL.
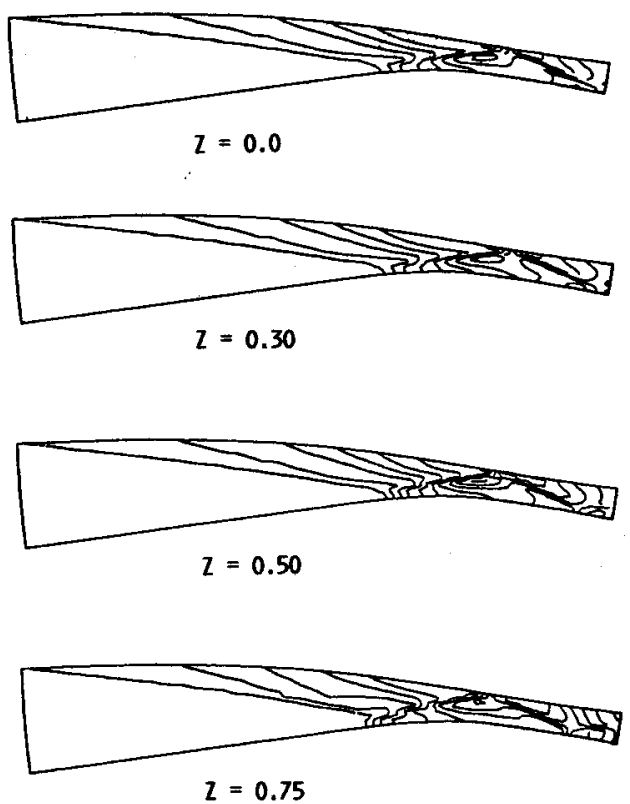

FIGURE 12.- STATIC PRESSURE CONTOURS OF P8 INLET AT VARIOUS SPAMNISE LOCATIONS. 


\section{ORIGINAL PAGE IS \\ OF POOR QUALT'}

$P_{p} / P_{1}$
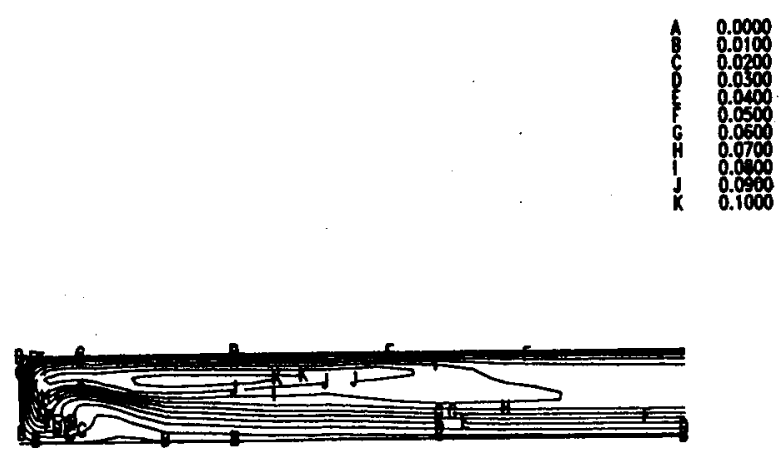

FIGURE 13.- PITOT PRESSURE CONTOUR MAP NEAR INLET EXIT.

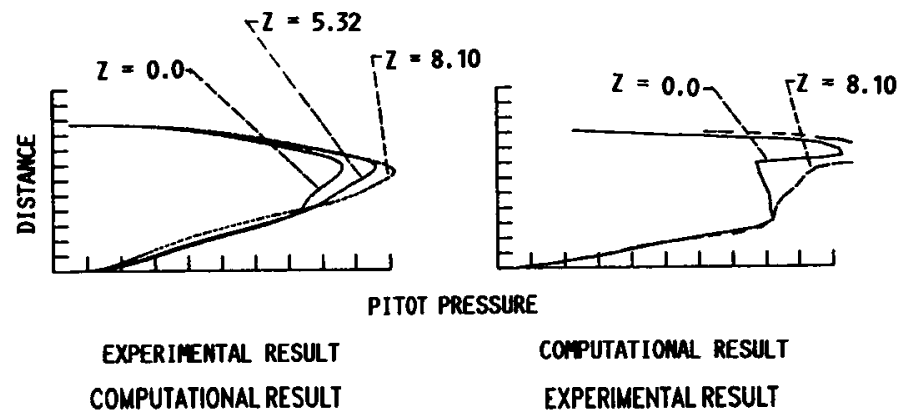

FIGURE 14. - COMPARISON OF PITOT PRESSURE PROFILES AT THE THROAT OF THE IMLET FOR VARIOUS LATERAL LOCATIONS. 


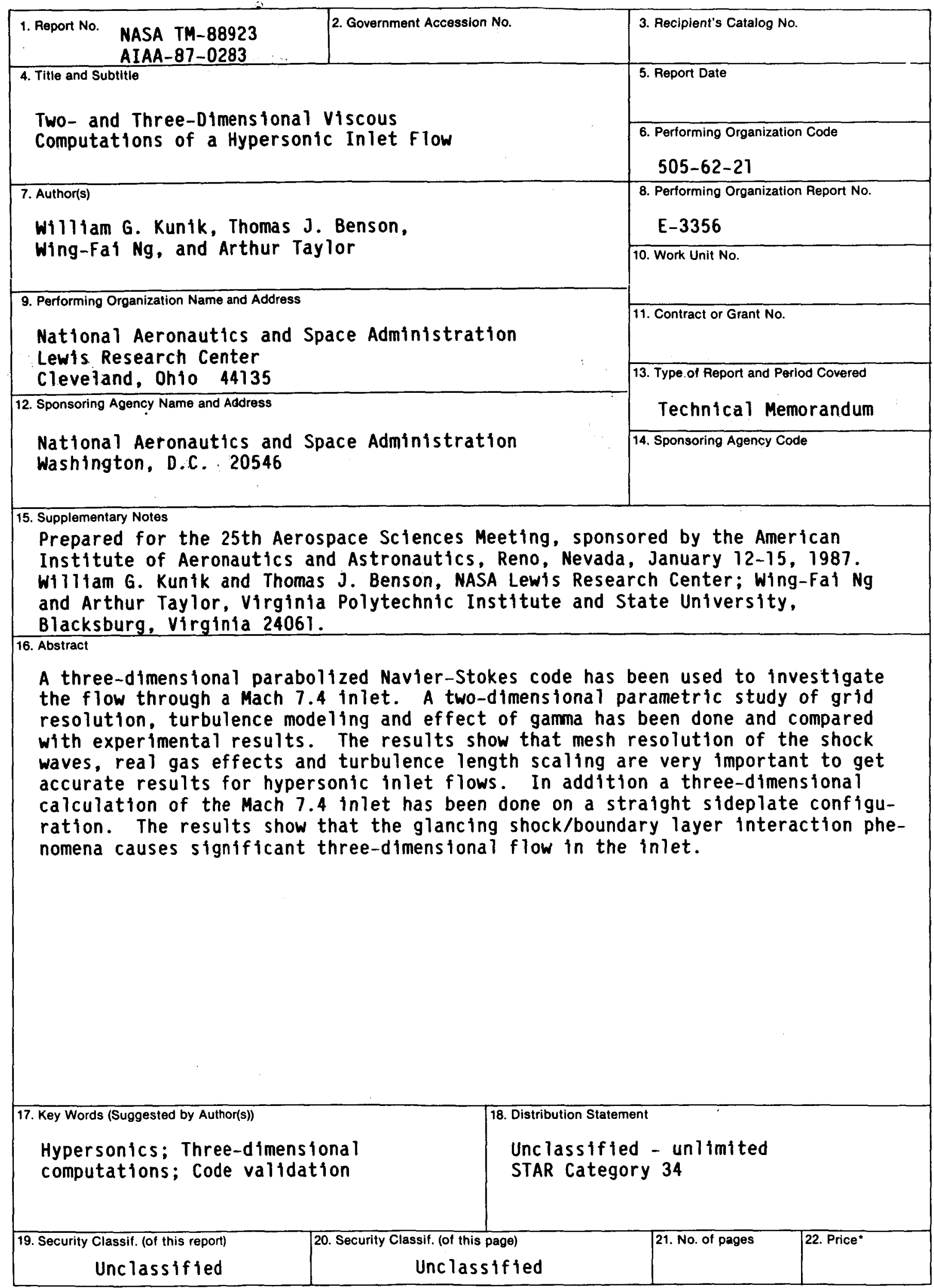

"For sale by the National Technical Information Service. Springfield, Virginia 22161 